\title{
Comparison of laparoscopic cystectomy and purse-string suture technique in the surgical treatment of ovarian dermoid cysts
}

\author{
(1) Remzi Atilgan, (1) Şehmus Pala \\ Department of Obstetrics and Gynecology, Fırat University Faculty of Medicine, Elazığ, Turkey
}

\begin{abstract}
Introduction: This study is a comparison of the outcomes of 2 cystectomy modalities in the surgical treatment of ovarian dermoid cysts.

Materials and Methods: The intraoperative and postoperative results of 44 ovarian dermoid cyst patients who underwent surgical treatment between 2012 and 2019 using 2 different methods were compared. The study population was divided into 2 groups: Group $1(n=21)$ underwent cystectomy using the purse-string suture method via mini-laparotomy and Group $2(n=23)$ underwent laparoscopic cystectomy.

Results: The mean ovarian cyst diameter was $11 \mathrm{~cm}$ in Group 1 and $6 \mathrm{~cm}$ in Group 2. The diameter of the cyst was significantly larger in Group 1 than in Group 2. The duration of the operation was significantly shorter in Group 1 than Group 2 (45 $\mathrm{min}$. vs. $70 \mathrm{~min}$.). The intraoperative rupture rate was significantly lower in Group 1 compared with Group 2 (4.7\% vs. 30\%). Chemical peritonitis did not develop in either group. The duration of hospitalization was similar between the groups. The recurrence rate was significantly lower in Group 1 than Group 2 (0\% vs. $21 \%$ ).

Conclusion: For clinicians who do not have the optimal conditions or surgical experience for a laparoscopic operation, and particularly for large ovarian dermoid cysts, the cystectomy technique performed using a purse-string suture via a mini-Pfannenstiel incision can be an effective treatment method.

Keywords: Laparoscopy; mini-Pfannenstiel incision; ovarian dermoid cyst; purse-string suture.
\end{abstract}

\section{Introduction}

Dermoid cysts are the most common germ cell ovarian tumor developing in the second and third decades of life. ${ }^{[1]}$ Dermoid cysts with a giant size $(>15 \mathrm{~cm})$ are very rare and may present with torsion or rupture. ${ }^{[2]}$ Intra-abdominal bleeding is the acute complication of rupture; however, intensive adhesion due to significant granulomatous reaction (chemical peritonitis) is the chronic complication of rupture. ${ }^{[3]}$
Choice of treatment methods for ovarian dermoid cysts varies among gynecologists. Various approaches and procedures were applied. Continuous improvement in laparoscopy allowed us to consider endoscopy a safe and effective alternative to laparotomy. ${ }^{[4]}$ Significantly long operation time and high cystic component spillage rate are the two main disadvantages of laparoscopic cystectomy procedure. ${ }^{[5]}$ It is reported that laparoscopy causes less side effects and less postoperative pain with shorter 
duration of hospital stay, better panoramic imaging, and significantly lower cost. ${ }^{[4]}$ Today, it is considered that laparoscopy is more favorable than laparotomy. ${ }^{[6]}$ Therefore, the present study aims to compare the intraoperative and postoperative outcomes of dermoid cyst patients underwent laparoscopy or purse-string suture operation with mini-laparotomy.

\section{Materials and Methods}

This study was designed as retrospective study. The study population included patients who were treated for a malignancy by radiological, biochemical, and physical examination and preoperatively diagnosed with dermoid cyst between 2012 and 2019 in Obstetrics and Gynecology Clinic of Frrat University Hospital. The results of 21 patients (Group 1, $\mathrm{n}=21$ ) who underwent an operation using the purse-string suture with mini-Pfannenstiel-assisted cystectomy, ${ }^{[7]}$ (size over $8 \mathrm{~cm}$ ) and the results of 23 patients (Group 2, n=23) who were treated with laparoscopic cystectomy (size below $10 \mathrm{~cm}$ ) were presented. Information obtained from the records of the patients, such as age, parity, duration of the operation and hospitalization, ovarian dermoid cyst diameters, rupture complication during the operation, amount of bleeding were compared. Ultrasound and magnetic resonance imaging techniques were used to diagnose dermoid cysts in all patients.

\section{Purse-string Suture with Mini-Pfannenstiel-Assisted CystectomyTechnique}

Under general anesthesia, a mini-Pfannenstiel incision of approximately 3-4 cm was made to the abdomen. After, a round suture line (purse-string suture), which was at about $1 \mathrm{~cm}$ diameter, was formed with $1 / 0$ vicryl as delicate as possible not to lead to cyst explosion. After this suture plan was created, the two ends of the rope were raised upwards by applying mild traction (Fig. 1). Then, the edges of the cyst were wrapped with a roll to prevent any cyst material escaping into the abdomen. Then the cyst wall was cut with a bistoury and immediately the aspirator hose was inserted into the cyst and the aspirator hose was squeezed by pulling two ends of the rope that was in our hands. The content of the cyst was thoroughly aspirated (Fig. 2). By holding from the open sides of the cyst with two clamps, the cyst was anastomosed outwards. After the compact materials in the cyst and the dense hair balls were completely cleaned using a forceps, the cyst and ovarian tissue could be removed out easily (Fig. 3). Then cystectomy was performed in a way to give

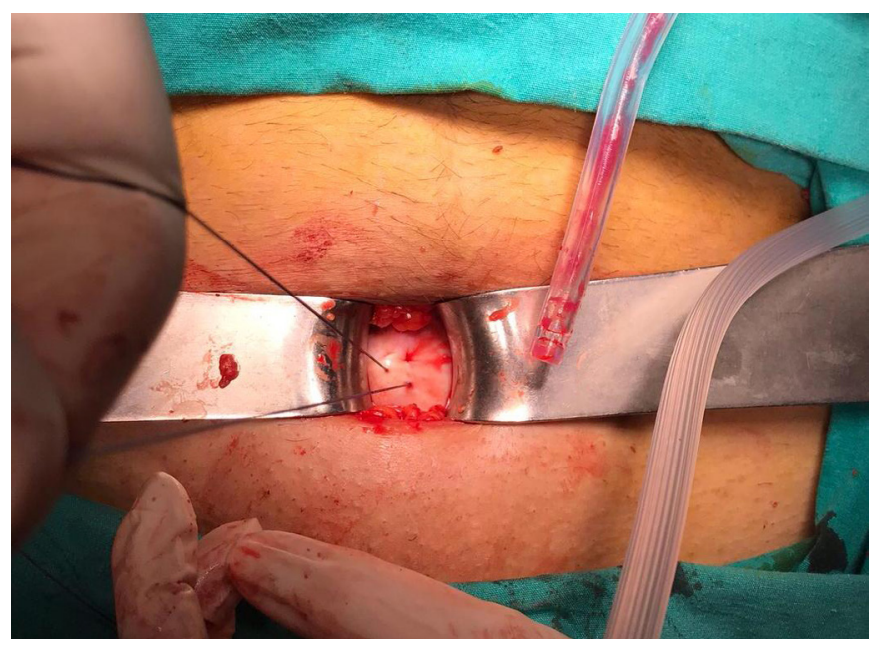

Figure 1. Mini-Pfannenstiel incision and placement of purse string suture is shown.

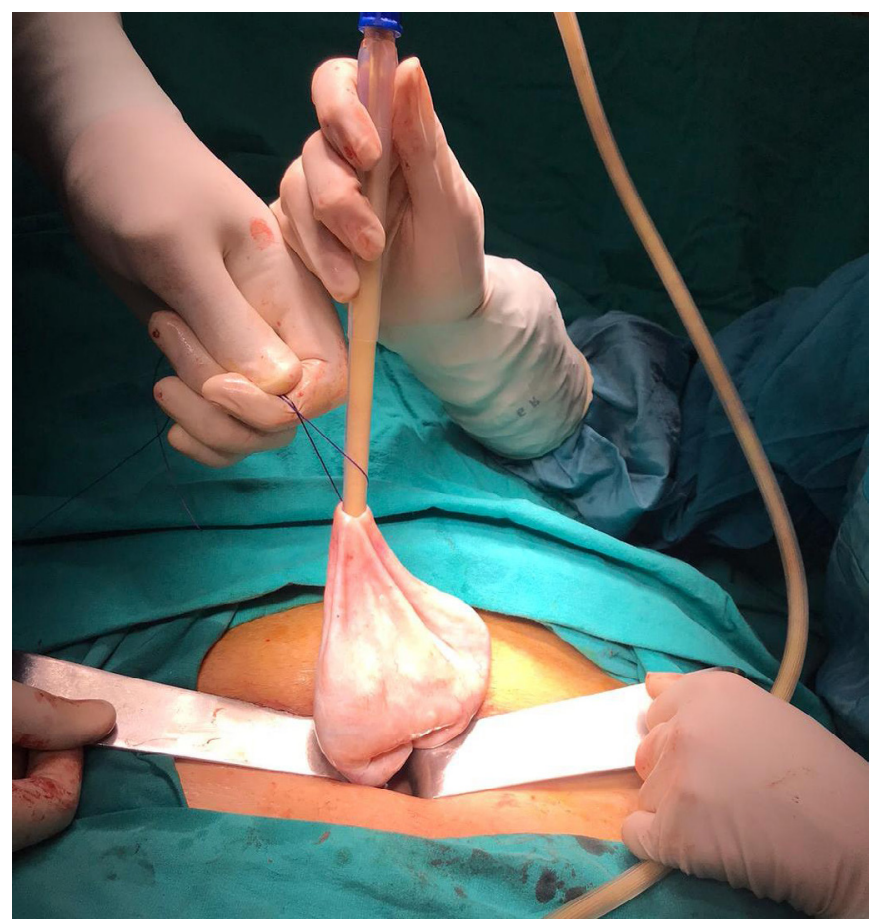

Figure 2. Aspiration of cyst contents outside the abdomen is shown.

minimal damage to the ovarian tissue and hemostasis was ensured. Then the ovary was put in the abdomen and the layers of the abdomen and skin were closed (Fig. 4).

\section{Laparoscopic Cystectomy Technique}

Under general anesthesia, approximately $1 \mathrm{~cm}$ vertical incision was to the site under the umbilicus. The abdomen was inflated with gas insufflation via Veress needle until pneumoperitoneum was formed. From the same incision, a 10-mm trocar was introduced to the abdomen and zero or 30-degree of the telescope was sent to the abdomen. 


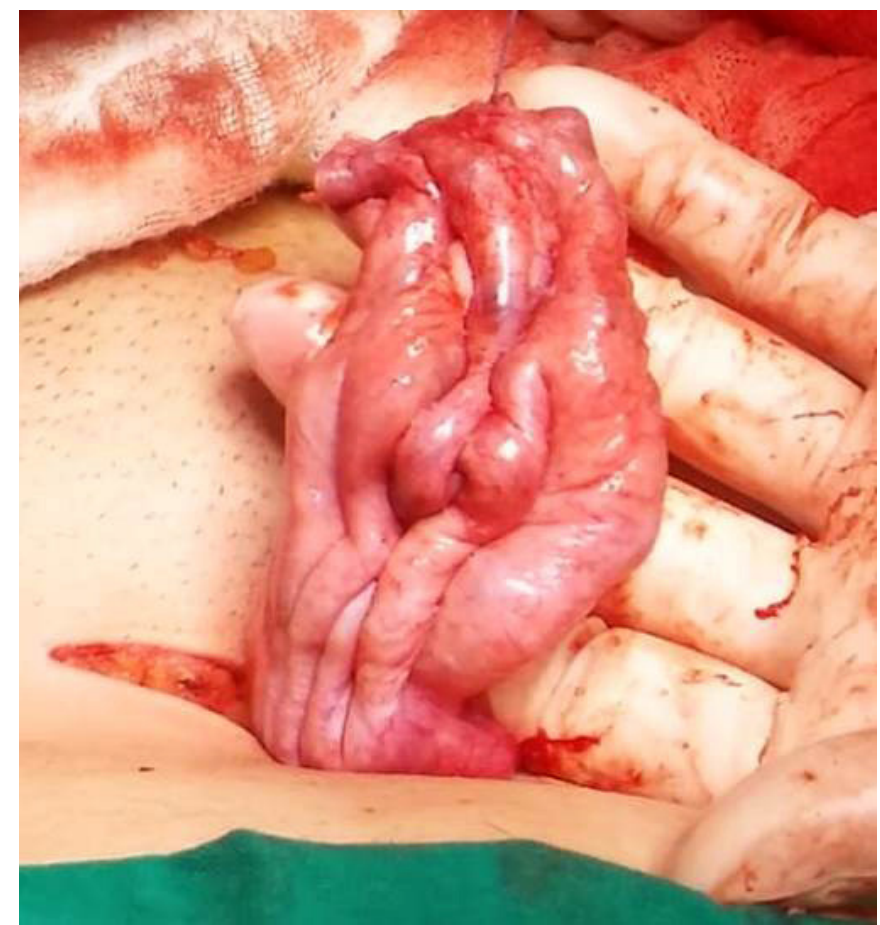

Figure 3. Postoperative ovarian view.

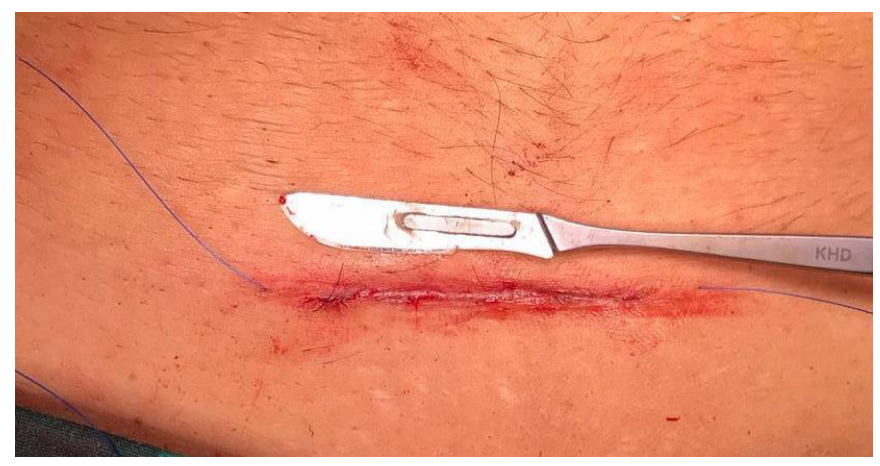

Figure 4. Postoperative view of mini-Pfannenstiel incision is shown.

The patient was positioned to the Trendelenburg. After all abdomen exploration was performed, the abdomen was entered with two ipsilateral and, in some patients, with one contralateral 5-mm trocar. For the bilaterally trocar introductions, the area at about $2 \mathrm{~cm}$ medial of the crista iliaca anterior superior without any veins was selected. The ipsilateral trocar entry was performed on the side determined by the surgeon, which was in the same line with the previous trocar and at approximately $6-7 \mathrm{~cm}$ superior to it. In the operations, monopolar electro coagulation and bipolar electro coagulation (LigaSure, Covidien Company, USA or ENSEAL, Ethicon Company, USA) were used as energy modality. Then, with the help of hydrostatic pressure and scissors, without causing any rupture in the cyst, it was attempted to resection the ovarian tissue together with the cyst capsule. Excised part via cystectomy was punctured in an endobag and the materials were removed from the umbilical incision. Later, hemostasis was ensured by monopolar and bipolar electrocoagulation in a way to cause minimal damage to the ovarian tissue and the operation was terminated following the bleeding control and abdominal irrigation in ruptured patients. The study protocol was approved by the Ethics Committee of Firat University (Date: 21.02.2019, Session no: 2019/04, Decision no: 21).

\section{Statistical Analysis}

The data were analyzed using the SPSS version 21.0 (IBM Corporation, Armonk, NY, USA) software. The distribution of continuous variables was determined using Kolmogorov-Smirnov normality test. Nonparametric or non-normal distribution of numerical data was expressed in median (minimum-maximum). Comparison of the binary variables that did not meet the normal distribution or non-parametric variables was performed using the Mann-Whitney-U test. Chi-square or Fisher exact test was used in the analysis of categorical data. A p value of $<0.05$ was considered statistically significant.

\section{Results}

The pathology results of all cases were reported as benign. There was no difference in age, gravida, and duration of hospitalization between the groups. The diameter of the cyst was significantly larger in Group 1 than Group $2(11 \mathrm{~cm}$ vs. $6 \mathrm{~cm}, \mathrm{p}=0.000)$. The duration of the operation was significantly shorter in Group 1 than Group 2 (45 min. vs. 70 min., $p=0.000$ ). The rupture rate was significantly lower in Group 1 compared to Group 2 during the operation ( $4.8 \%$ vs. $30 \%, p=0.048)$. Chemical peritonitis did not develop in both groups. The duration of hospitalization was similar between the groups ( 36 hours in Group 1 and 36 hours in Group 2) (Table 1).

\section{Discussion}

The Royal College of Obstetricians and Gynecologists (RCOG) recommend that when an operation is required, a laparoscopic approach should be generally considered gold standard for the treatment of benign ovarian masses. Laparotomy may be appropriate in the presence of large masses having solid components such as large mature cystic teratoma. However, maximum ovarian cyst size for laparotomy is controversial. ${ }^{[8]}$ Some researchers suggest laparotomy for mature cystic teratomas above $10 \mathrm{~cm} .{ }^{[9]} \mathrm{In}$ addition, RCOG recommends a laparoscopic surgery per- 
Table 1. The age, parity, intraoperative and postoperative results in both groups

\begin{tabular}{lccc} 
Parameters & $\begin{array}{c}\text { G1 }(\mathbf{n}=\mathbf{2 1}) \\
\text { Median (Min-Max) }\end{array}$ & $\begin{array}{c}\text { G2 }(\mathbf{n}=\mathbf{2 3}) \\
\text { Median (Min-Max) }\end{array}$ & p \\
\hline Age (years) & $29(16-42)$ & $30(16-41)$ & 0.284 \\
Parity & $2(0-4)$ & $2(0-4)$ & 0.971 \\
Operation time (minutes) & $45(40-90)$ & $70(40-120)$ & $0.000^{*}$ \\
Intraoperetaive rupture & 1 case & 7 case & $0.048^{*}$ \\
Bleeding amount (cc) & $100(100-250)$ & $100(50-200)$ & 0.816 \\
Cyst diameters (cm) & $11(8-17)$ & $6(4-10)$ & $0.000^{*}$ \\
Duration of hospital stay (hours) & $36(24-60)$ & $36(24-96)$ & 0.909 \\
\hline$*=$ p<0.05 was considered statistically significant. & & &
\end{tabular}

formed by a surgeon, who has sufficient experience, with appropriate instruments. Appropriate surgical treatment of ovarian masses depends on a variety of factors including female sex (including compliance with laparoscopy and her wishes), mass (size, complexity and possible quality), and environment (including surgeon's skills and instruments). Considering these factors, a decision must be made after careful clinical evaluation and consultation. ${ }^{[8,9]}$ In this study, these factor were also taken into consideration in the choice of surgical procedure. In our patients with dermoid cyst at a diameter of $>8 \mathrm{~cm}$, a cystectomy was performed using purse-string suture method with mini-Pfannenstiel incision.

Laberge and Levesque ${ }^{[10]}$ reported that laparoscopic cystectomy significantly had a longer duration of operation and a higher rate of spillage of cyst content ( $1 \%$ versus $18 \%$ ) when compared the laparoscopic approach with laparotomy. In the case of spillage of the ovarian dermoid cyst contents, chemical peritonitis is rare but its treatment is difficult. In different studies, this situation was reported to occur in less than $0.2 \%$ of patients. ${ }^{[1]}$ The spillage of content is significantly higher in laparoscopic approach than the approach with laparotomy. ${ }^{[10,12-14]}$ There are also studies reporting that this rate is $100 \% \cdot{ }^{[15]}$ In our study, chemical peritonitis did not develop in any of the patients. Intraoperative cyst rupture rate (31\% vs. $4 \%)$ and operation period (70 min. vs. $45 \mathrm{~min}$.) were higher in laparoscopy compared to the purse-string suture method.

The conventional treatment of giant ovarian cysts is usually a complete midline laparotomy. This is followed by a cystectomy or oophorectomy. ${ }^{[2,16]}$ Midline vertical incision laparotomy and an ovarian loss resulting from this procedure may reduce patient's fertility capacity and lead to a visible vertical scar on the skin. The results of this treatment are not acceptable for many young and nulliparous patients. ${ }^{[17]}$ In our cases, visible incision scar was minimal.

In a surgery performed using open laparoscopic technique, Lee et al. ${ }^{[18]}$ aspirated the contents of the cyst using the purse-string suture method and continued with laparoscopy. They called this method as "laparoscopicallyassisted extracorporeal cystectomy". Li et al. ${ }^{[19]}$ described a combined mini-laparotomy and laparoscopy method in the surgical treatment of large ovarian masses. While minilaparotomy incision was used to minimize ovarian mass and remove the specimen in this method, laparoscopy was performed in the rest of the procedure. Especially in patients with dermoid cyst at a diameter of $>8 \mathrm{~cm}$ and in which radiologically intensive content was detected, we also performed open cystectomy with mini-Pfannenstiel incision to avoid the possibility of spilling of cyst contents into the abdomen as laparoscopic surgery might be difficult. There was no significant cyst fluid spillage in any of the patients. We also used the purse-string suture method like Lee et al. ${ }^{[18]}$ However, the difference of our implementation from that of Lee et al. was that we performed the mini-Pfannenstiel incision instead of the midline incision which was used for open laparoscopy.

The vast majority of surgical procedures in the world are shifting towards minimally invasive methods. We cannot be out of this technique. In our clinic, we use laparoscopy as the first choice in all cystectomy operations we consider benign. However, open cystectomy may be planned in the absence of adequate surgical equipment and experienced laparoscopic surgeon. In this study, we aimed to encourage the surgeon to use a rather small mini-Pfannenstiel incision instead of a large Pfannenstiel incision in cases 
where cystectomy is planned to be performed with at least open surgery. Compared to a large Pfannenstiel incision, the mini-Pfannenstiel incision can also be considered a minimally invasive method. In order to reduce the possible rupture complication during cyst excision with laparoscopy, an endobag to be kept under the cyst with an additional port input can prevent the contents from spreading to the abdomen during possible rupture.

In this study, it was observed that the possibility of cyst rupture increased with laparoscopic surgery. Moreover, it became difficult to remove the cyst content including bone pieces and hair balls larger than the umbilical port diameter from the umbilical incision.

There are also some limitations to the present study. It was a retrospective study and the data on the applicability of the method in obese patients were limited. Furthermore, dissection of the cyst may be difficult with this incision in adherent cases.

In conclusion, laparoscopy is the gold standard method in the treatment of benign ovarian cysts. Especially for clinicians who do not have optimal conditions for laparoscopic surgical experience and laparoscopic operation, the cystectomy technique performed using purse-string suture via mini-Pfannenstiel incision may be an effective treatment method for large ovarian dermoid cysts.

\section{Disclosures}

Ethichs Committee Approval: The study protocol was approved by the Ethics Committee of Firat University (Date: 21.02.2019, Session no: 2019/04, Decision no: 21).

Peer-review: Externally peer-reviewed.

Conflict of Interest: None declared.

Authorship Contributions: Concept - R.A.; Design R.A.; Supervision - R.A.; Materials - Ş.P.; Data collection and/or processing - S.P.; Analysis and/or interpretation - Ş.P.; Literature search - Ş.P.; Writing - R.A.; Critical review - R.A.

\section{References}

1. Shalev E, Bustan M, Romano S, Goldberg Y, Ben-Shlomo I. Laparoscopic resection of ovarian benign cystic teratomas: experience with 84 cases. Hum Reprod 1998;13:1810-2.

2. Dolan MS, Boulanger SC, Salameh JR. Laparoscopic management of giant ovarian cyst. JSLS 2006;10:254-6.

3. Esmaeili M, Shahbazi S, Seylani K, Nazari N. Ovarian dermoid cyst and neuromuscular manifestation. Nurs Pract Today
2015;2:171-73.

4. Sinha A, Ewies AA. Ovarian Mature Cystic Teratoma: Challenges of Surgical Management. Obstet Gynecol Int 2016;2016:2390178. [CrossRef]

5. Alborzi S, Foroughinia L, Kumar PV, Asadi N, Alborzi S. A comparison of histopathologic findings of ovarian tissue inadvertently excised with endometrioma and other kinds of benign ovarian cyst in patients undergoing laparoscopy versus laparotomy. Fertil Steril 2009;92:2004-7. [CrossRef]

6. Matsushita $\mathrm{H}$, Watanabe $\mathrm{K}$, Yokoi T, Wakatsuki A. Unexpected ovarian malignancy following laparoscopic excision of adnexal masses. Hum Reprod 2014;29:1912-7. [CrossRef]

7. Atilgan R, Pala Ş, Bulu G. Purse-string suture technique using a mini-Pfannenstiel incision to treat large dermoid cysts. J Obstet Gynaecol. 2020; 8:1-4. [CrossRef]

8. Royal College of Obstetricians and Gynaecologists (RCOG). Management of Adnexal Masses in Premenopausal Women. London, UK: Royal College of Obstetricians and Gynaecologists (RCOG); 2011.

9. Chang CK, Teng SW, Leu FJ. Laparoscopy versus laparotomy for cystic ovarian teratomas. Int J Gynaecol Obstet 2005;88:69-70. [CrossRef]

10. Laberge PY, Levesque S. Short-term morbidity and longterm recurrence rate of ovarian dermoid cysts treated by laparoscopy versus laparotomy. J Obstet Gynaecol Can 2006;28:789-93. [CrossRef]

11. American College of Obstetricians and Gynecologists. ACOG Practice Bulletin. Management of adnexal masses. Obstet Gynecol 2007;110:201-14. [CrossRef]

12. Benezra V, Verma U, Whitted RW. Comparison of laparoscopy versus laparotomy for the surgical treatment of ovarian dermoid cysts. Gynecological Surgery 2005;2:89-92. [CrossRef]

13. Kondo W, Bourdel N, Cotte B, Tran X, Botchorishvili R, Jardon $K$, et al. Does prevention of intraperitoneal spillage when removing a dermoid cyst prevent granulomatous peritonitis? BJOG 2010;117:1027-30. [CrossRef]

14. Kruschinski D, Homburg S, Langde S, Kapur A. Dermoid tumors of the ovary: evaluation of the gasless lift-laparoscopic approach. Surg Technol Int 2008;17:203-7.

15. Savasi I, Lacy JA, Gerstle JT, Stephens D, Kives S, Allen L. Management of ovarian dermoid cysts in the pediatric and adolescent population. J Pediatr Adolesc Gynecol 2009;22(6):360-4. [CrossRef]

16. Mülayim B, Gürakan H, Dagli V, Mülayim S, Aydin O, Akkaya H. Unaware of a giant serous cyst adenoma: a case report. Arch Gynecol Obstet 2006;273:381-3. [CrossRef]

17. Uyanikoglu H, Dusak A. A Huge Ovarian Dermoid Cyst: Successful Laparoscopic Total Excision. J Clin Diagn Res 2017; 11:QD03-5. [CrossRef]

18. Lee LC, Sheu BC, Chou LY, Huang SC, Chang DY, Chang WC. An easy new approach to the laparoscopic treatment of large adnexal cysts. Minim Invasive Ther Allied Technol 2011;20:150-4. [CrossRef]

19. Li T, Tan J, Cohen P. A novel surgical technique for the large ovarian cystic mass - combined mini-laparotomy and laparoscopy. Eur J Gynaecol Oncol 2016;37:766-70. 